Pathologe 2018 · 39 (Suppl 2):S324 https://doi.org/10.1007/s00292-018-0481-3 Online publiziert: 17. August 2018 (c) Springer Medizin Verlag GmbH, ein Teil von Springer Nature 2018

CrossMark

\section{T. Braunschweig' $\cdot$ K. Schierle ${ }^{2}$}

'Institut für Pathologie, Uniklinik RWTH Aachen, Aachen, Deutschland

${ }^{2}$ Institut für Pathologie, Universitätsklinikum Leipzig, Leipzig, Deutschland

\title{
Sitzung der Arbeitsgemeinschaft Geschichte der Pathologie
}

\author{
102. Jahrestagung der Deutschen \\ Gesellschaft für Pathologie in Berlin
}

Inzwischen fand das dritte Treffen der Arbeitsgemeinschaft im Rahmen der 102. Jahrestagung der Deutschen Gesellschaft für Pathologie (DGP) statt, nachdem sie zur 100. Jahrestagung im Jahr 2016 gegründet wurde. Die Sitzung setzte sich aus einem Gastvortrag und vier weiteren Vorträgen als wissenschaftlicher Teil und einer Mitgliederversammlung zusammen.

Der Gastvortrag von D. Groß als Leiter des Instituts für Geschichte, Theorie und Ethik der Medizin in Aachen beleuchtete sehr anschaulich, mit welcher Quellenlage beim Projekt zur Pathologie im Nationalsozialismus $\mathrm{zu}$ rechnen ist und wie die Aufarbeitung gegliedert ist. Die Darlegung der Konzeption des Projektes erregte Aufmerksamkeit über die Arbeitsgruppe hinaus. Zusätzlich erfolgte eine weitere Erläuterung und Abgrenzung des Projekts der DGP zu den anderen, mit und durch den Bundesverband Deutscher Pathologen getragenen Projekte. Hier ist geplant, zum einen speziell die Repräsentanten der DGP (inkl. der von ihr geehrten Fachvertreter) und zum anderen „Opfer“ unter den Pathologen im Nationalsozialismus zu beleuchten, soweit dies mit den verfügbaren Quellen möglich ist. Im Anschluss folgte eine angeregte Diskussion, bei der aus dem Auditorium unter anderem auf die sanitätsakademische Sammlung der Bundeswehr in München als weitere Quelle aufmerksam gemacht wurde.

Nach dem Gastvortrag wurde eine Mitgliederversammlung der AG in das Programm eingeschoben. Hier wurden nochmals die verschiedenen Projekte zur Aufarbeitung der Pathologie im Natio- nalsozialismus dargelegt. Zudem ist noch zu klären, welchen Stellenwert die Geschichte der Pathologie im Programm der DGP-Jahrestagung 2019 haben wird. Hierfür werden weitere Gespräche mit dem designierten Kongresspräsidenten Herrn Prof. Schmid geführt werden. Es wird auch erörtert werden, ob es möglich ist, eine Serie von Publikationen in Der Pathologe zur Geschichte der Pathologie zu veröffentlichen. Aus dem Auditorium lagen keine weiteren Themenvorschläge vor.

Zwei der weiteren Vorträge stammten von S. Koch, der sich in einem Vortrag mit den physiologisch-chemischen Untersuchungen Virchows beschäftigte. Er kam zu dem Fazit, dass die angewandten, überwiegend chemischen Methoden der damaligen Zeit für Virchow keinen weiteren Erkenntnisgewinn brachten und auch in Versuchen selten kritisch diskutiert wurden. Der zweite Vortrag von S. Koch beleuchtete die Stilmittel der medizinischen Schriften Virchows, die sich durch hohe sprachliche und kommunikative Kompetenz, geprägt durch den von Virchow vertretenen naturwissenschaftlichen Ansatz, auszeichnen. M. von Haugwitz legte in seinem Vortrag das Leben von Allvar Gullstrand als Wegbereiter der Dioptrik und als Nobelpreisträger dar, dessen Wirken nur unter dem Verständnis höherer Mathematik möglich war. T. Braunschweig wählte in seinem Vortrag einen möglichen Ansatz für weitere Aufarbeitungen der wissenschaftlichen Arbeiten von 1920-1940. Es wurden Publikationen aus ausgewählten Journalen des deutschen und englischen Sprachraums hinsichtlich des Inhalts ausgewertet und es fällt auf, dass in dieser Zeit sehr viel publiziert wurde und einzelne Publikationen bzw. Publikationsthemen besonders hervorzuheben sind. In einigen Publikationen von Frauen konnten „Opfer“ des Nationalsozialismus gefunden werden, zur Unterstützung eines Projektes von Prof. Groß. Zum Zweiten finden sich bereits Artikel über Zellkultur und XenograftExperimente. Ungewöhnlich auch der stark bearbeitete Bereich der tierexperimentellen Forschung mit der Reaktion auf Biomaterialien. Hier wird sich weitere Forschung anschließen.

\section{Korrespondenzadresse}

Dr. T. Braunschweig

Institut für Pathologie, Uniklinik RWTH Aachen Pauwelsstr. 30, 52074 Aachen, Deutschland tbraunschweig@ukaachen.de

\section{Dr. K. Schierle}

Institut für Pathologie, Universitätsklinikum Leipzig

Liebigstr. 26, 04103 Leipzig, Deutschland

katrin.schierle@medizin.uni-leipzig.de

\section{Einhaltung ethischer Richtlinien}

Interessenkonflikt. T. Braunschweig und K. Schierle geben an, dass kein Interessenkonflikt besteht.

Dieser Beitrag beinhaltet keine von den Autoren durchgeführten Studien an Menschen oder Tieren.

The supplement containing this article is not sponsored by industry. 